\title{
D-D nuclear fusion processes induced in polyethylene foams by TW Laser-generated plasma
}

\author{
L. Torrisi ${ }^{1, a}$, M. Cutroneo ${ }^{2}$, S. Cavallaro ${ }^{1}$ and J. Ullschmied ${ }^{3}$ \\ ${ }^{1}$ Department of Physics and Earth Science, V.le S. d'Alcontres 31, 98166 S. Agata (ME), Italy \\ ${ }^{2}$ Institute of Nuclear Physics, ASCR-Rez, Czech Republic \\ ${ }^{3}$ Institute of Plasma Physics ASCR, 18200 Prague 8, Czech Republic
}

\begin{abstract}
Deuterium-Deuterium fusion processes were generated by focusing the 3 TW PALS Laser on solid deuterated polyethylene targets placed in vacuum. Deuterium ion acceleration of the order of $4 \mathrm{MeV}$ was obtained using laser irradiance $\mathrm{I} \lambda^{2} \sim 5 \times 10^{16} \mathrm{~W} \mu \mathrm{m}^{2} / \mathrm{cm}^{2}$ on the target. Thin and thick targets, at low and high density, were irradiated and plasma properties were monitored "on line" and "off line". The ion emission from plasma was monitored with Thomson Parabola Spectrometer, track detectors and ion collectors. Fast semiconductor detectors based on $\mathrm{SiC}$ and fast plastic scintillators, both employed in time-of-flight configuration, have permitted to detect the characteristic $3.0 \mathrm{MeV}$ protons and $2.45 \mathrm{MeV}$ neutrons emission from the nuclear fusion reactions. From massive absorbent targets we have evaluated the neutron flux by varying from negligible values up to about $5 \times 10^{7}$ neutrons per laser shot in the case of foams targets, indicating a reaction rate of the order of $10^{8}$ fusion events per laser shot using "advanced targets".
\end{abstract}

\section{Introduction}

The high intensity laser, of the order of $10^{16} \mathrm{~W} / \mathrm{cm}^{2}$ or higher, transfers high ponderomotive energy to the electrons target, generating high electron emission and high electric fields in the developed non equilibrium plasma, which drives the ion acceleration mainly along the normal to the target surface [1]. Thermal and Coulomb interactions and hydrodynamical pressures contribute to the final ion velocity showing energy from hundred $\mathrm{keV} /$ charge state to values above $10 \mathrm{MeV} /$ charge state. Particularly interesting appears the acceleration of protons both in backward plasma acceleration (BPA), using thick targets, and Target Normal Sheath Acceleration (TNSA), using thin targets regime [2]. In the first case high ion yields but low ion kinetic energies are obtainable, while in the second case low ion yields and high ion kinetic energies are measurable. At very high intensity the ion acceleration is enhanced by the radiation pressure acceleration (RPA) developed on very thin foils of the order of 10-100 nm in thickness [3].

Accelerated ions generally exhibit a Boltzmann-like distribution with a cut-off at high energy and follow the Coulomb-Boltzmann-shifted distribution, increasing the ion energy proportionally to their charge state [4].

In the last years at PALS laboratory in Prague, releasing $300 \mathrm{ps}$ laser pulse duration, $1315 \mathrm{~nm}$ wavelength, 70 $\mu \mathrm{m}$ spot diameter and $10^{15} \mathrm{~W} / \mathrm{cm}^{2}$ intensity, have been accelerated deuterons using both BPA and TNSA regimes irradiating deuterated polyethylene targets [5]. Different di-

${ }^{\mathrm{a}}$ Corresponding Author: Lorenzo.Torrisi@unime.it agnostic techniques were employed to measure the energy of accelerated deuterons and of the products with characteristic energy coming from the D-D nuclear fusions, according to the two reaction channels having the same occurrence probability:

$$
\begin{aligned}
& \mathrm{D}+\mathrm{D} \rightarrow \mathrm{T}(1.01 \mathrm{MeV})+\mathrm{p}(3.02 \mathrm{MeV}) \\
& \mathrm{D}+\mathrm{D} \rightarrow{ }^{3} \mathrm{He}(0.82 \mathrm{MeV})+\mathrm{n}(2.45 \mathrm{MeV})
\end{aligned}
$$

Fig. 1 shows the plot of the D-D nuclear fusion crosssection vs. the deuterium ion energy. The maximum cross section occurs at $3.0 \mathrm{MeV}$ projectile energy at which its value is about 0.2 barns [6].

The use of thin targets in TNSA regime permits the occurrence of high ion acceleration in forward direction, from the rear side of the target, above $1 \mathrm{MeV}$ per charge state, but low yield is produced due to the limited thickness of the target. The use of thick targets permits to generate backward ions in BPA regime with a high yield, but with a low energy, generally below $100 \mathrm{keV}$ per charge state. The use of thick "advanced targets", promoting the laser absorption and the ion acceleration, for example based on porous (foam) polymers, permits to enhance both ion energy and ion yield accelerating protons at energies of the order of some MeVs. Tailored foams, due to their low density (approximately $1-10 \mathrm{mg} / \mathrm{cm}^{3}$ ) and high porosity, can be employed as thick and thin films covering a substrate in order to enhance the laser illuminated area, to reduce the reflection component of the laser light, to increase the laser penetration in the under-critical produced plasma and 


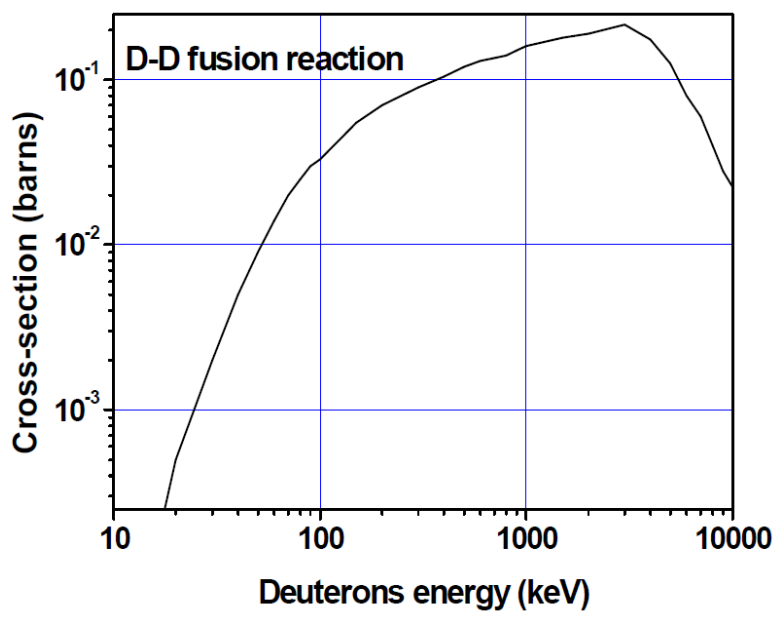

Figure 1. D-D cross-section vs. deuteron energy.

to induce self-focusing effect due to the high refraction index in the produced pre-plasma vapor [7].

\section{Experimental section}

The Asterix Iodine laser at PALS laboratory in Prague (Czech Republic) was employed for this experiment. Laser operates at $1315 \mathrm{~nm}$ wavelength, $700 \mathrm{~J}$ maximum pulse energy, 300 ps pulse duration, $70 \mu \mathrm{m}$ laser spot diameter, $10^{16} \mathrm{~W} / \mathrm{cm}^{2}$ pulse intensity and single pulse mode. Targets were based on thick and thin films of deuterated $\left(\mathrm{CD}_{2}\right)_{n}$ polyethylene $(\mathrm{PE})$, at high density $\left(0.93 \mathrm{~g} / \mathrm{cm}^{3}\right)$ as well as at very low density $\left(9 \mathrm{mg} / \mathrm{cm}^{3}\right)$, under shape of foams or porous material. The low foam density made them excellent as optical light absorbers. The polyethylene foam was obtained by physical and chemical processes inducing chain cross-linking in heated gas phase and high micrometric porosity. The used target thickness ranges between $5 \mu \mathrm{m}$ and $5 \mathrm{~mm}$.

A Thomson Parabola Spectrometer (TPS), coupled to a Multi-Channel-Plate (MCP), a phosphorous screen and a CCD camera, was placed in forward direction, along the normal to the target surface $\left(0^{\circ}\right)$, in order to measure the ion mass-to-charge ratio and energies emitted from plasma in single laser shots. TPS uses a deflecting magnetic and electric field of $0.01 \mathrm{~T}$ and $1 \mathrm{kV} / \mathrm{cm}$, respectively. Tosca 3D Code [8] was employed to calculate the magnetic and electric deflections and to simulate the ion parabolas using the real geometry and configurations of the used TPS. Simulations were compared with experimental data to ensure a good recognition of the parabola lines and an evaluation of the maximum ion energy. The laser-generated plasma was also monitored with Ion Collectors (IC), SiC detectors and plastic scintillators (NE-102A) employed in time-of-flight configuration and placed at known distances (60-120 $\mathrm{cm}$ for $\mathrm{SiC}$ and $120-240 \mathrm{~cm}$ for scintillators) from the target and at known angles $\left(0^{\circ}-170^{\circ}\right)$ from the target normal direction, using a fast storage oscilloscope. In particular IC is under vacuum and gives signals proportional the ion charge states and currents, $\mathrm{SiC}$ is under vacuum
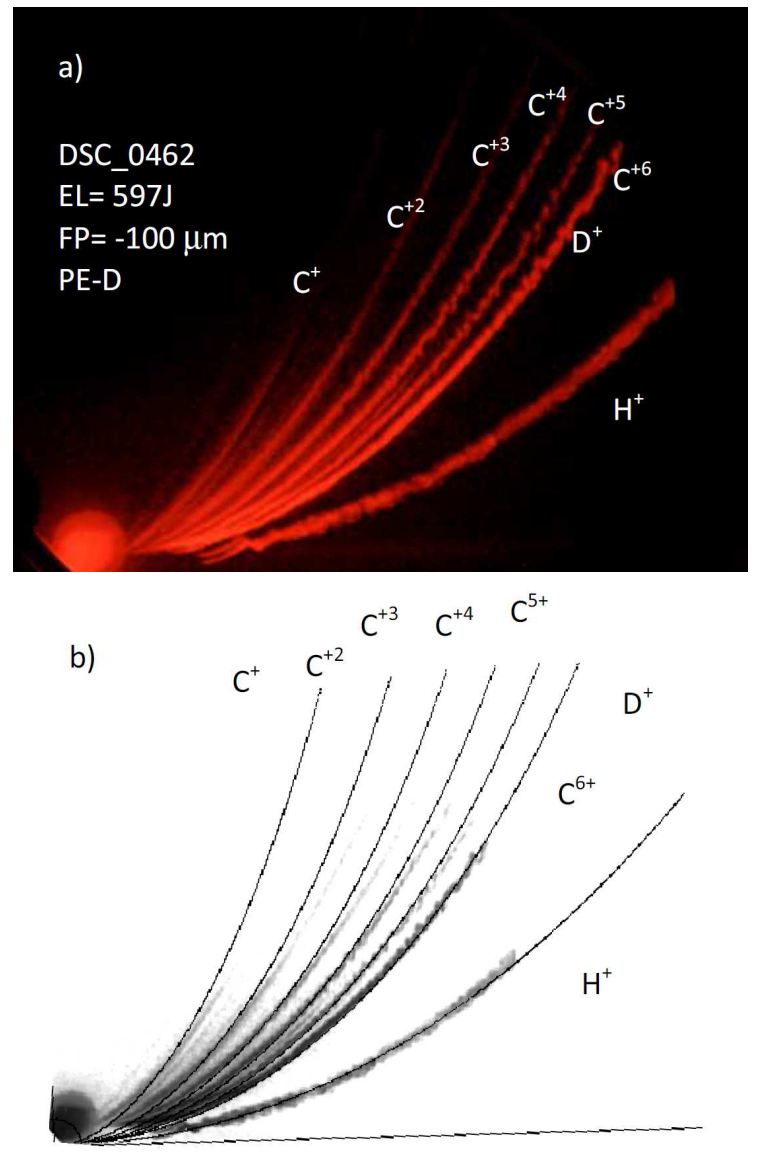

Figure 2. Typical TPS sectrum of $10 \mu \mathrm{m}$ deuterated PE (a) and overlap between spectrum and simulation data (b).

and has an input windows constituted by $200 \mathrm{~nm} \mathrm{Ni}_{2} \mathrm{Si}$ metallic electrode and a depletion layer of $80 \mu \mathrm{m}$ depth, instead the plastic scintillators are in air, out of the vacuum chamber, and are coupled to a fast high-gain photomultiplier tube, thus they are sensitive only to the gamma rays and to neutrons. A 2 ns exposition time X-ray CCD streak camera was placed on the top of the vacuum chamber, orthogonally to the target normal direction, in order to observe, though $50 \mu \mathrm{m}$ pin-hole diameter, the plasma emission from the target surface. Further details on the fast detection methods employed for plasma diagnostics are provided in the literature $[9,10]$. Micrometric stepmotors allowed a fine adjustment of the laser focal position (FP) with respect to the target surface position.

Moreover, track detectors using CR-39 films covered with different thicknesses of aluminum foil and calibrated bubble neutron dosimeters, with a sensitivity of 4 bubbles $/ \mu \mathrm{Sv}$ (Bubble Technology Industries, Chalk River, Ontario, Canada [11]), were employed to detect characteristic protons and neutrons emitted from the nuclear fusion processes. Both detectors have zero gamma sensitivity, require no power, and have an isotropic angular response. 

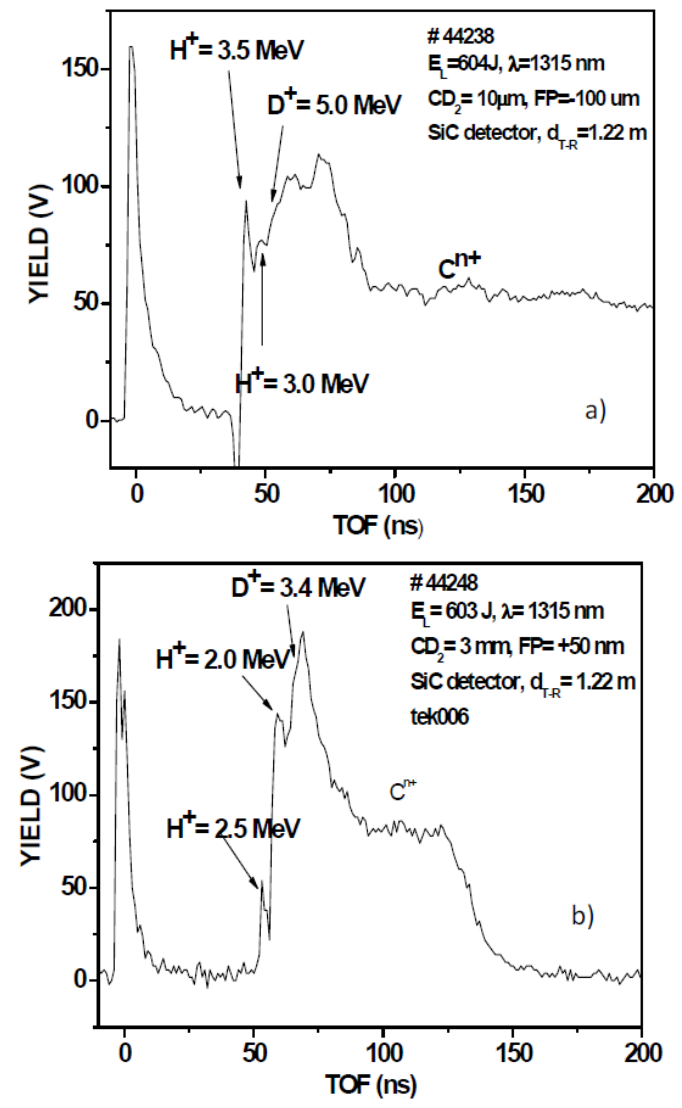

Figure 3. Typical $\mathrm{SiC}$ forward spectrum from TNSA irradiation of deuterated PE relative to the $10 \mu \mathrm{m}$ high density foil (a) and to $3 \mathrm{~mm}$ low density foam (b).

\section{Results}

A typical result obtained using the Thomson Parabola Spectrometer is reported in Fig. 2 for the TNSA plasma production, from $600 \mathrm{~J}$ pulse energy hitting a deuterated PE foil, $10 \mu \mathrm{m}$ in thickness, using a laser focal position FP of $-100 \mu \mathrm{m}$, i.e. focusing $100 \mu \mathrm{m}$ in front of the target surface. The spectrum (a) compared to the simulation data (b) permits to identify the protons and the six charge states of the carbon ions. The deuterium parabola is coincident with the $\mathrm{C}^{6+}$ parabola due to the same charge-to-mass ratio; thus this parabola has a higher light intensity with respect to the other $\mathrm{C}$ ion parabolas. The proton parabola is thicker and wider with respect to the others as a consequence of two proton contributions: the protons coming from the D-D nuclear reaction extended up to a maximum energy of $3.0 \mathrm{MeV}$ and the protons coming from the ionized hydrogen absorbed in the deuterated PE polymer, characterized by a maximum ion acceleration of $2.4 \mathrm{MeV}$. Fig. $2 b$ shows the overlapping between the simulation and the experimental parabolas obtained using the Tosca 3D Code from which it is possible to calculate a maximum energy of the less deflected ions which is $3.0 \mathrm{MeV}$ and 2.4 $\mathrm{MeV}$ for protons, 4.8 MeV for deuterium ions and about $2.4 \mathrm{MeV}$ per charge state for the $\mathrm{C}$ ions.

Typical $\mathrm{SiC}$ forward spectrum obtained in the case of TNSA plasma production from $10 \mu \mathrm{m}$ deuterated PE $(0.93$

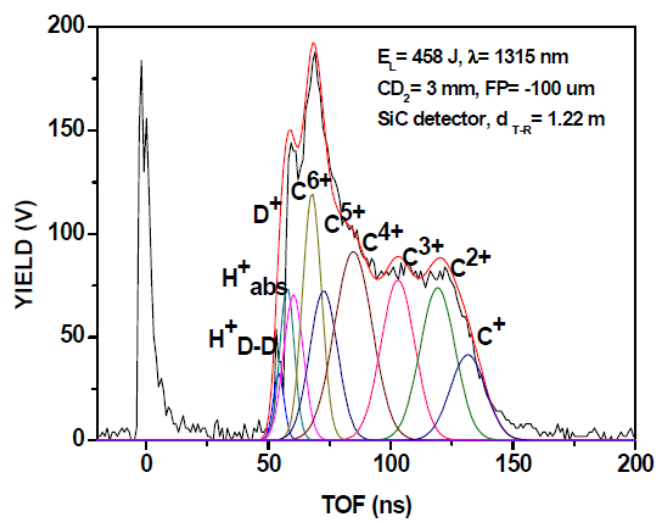

Figure 4. Deconvolution of a $\mathrm{SiC}$ spectrum to distinguish the different ion contributions using Boltzmann ion energy distributions.

$\mathrm{g} / \mathrm{cm}^{3}$ density) is reported in Fig. 3a, while a typical spectrum obtained by irradiating a thick $(3 \mathrm{~mm})$ low density deuterated PE foam ( $9 \mathrm{mg} / \mathrm{cm}^{3}$ density) is reported in Fig. 3b. In the first case the maximum proton energy is 3.5 $\mathrm{MeV}$ (coming from hydrogen absorbed in the polymer) and the maximum deuteron energy is $5.0 \mathrm{MeV}$. In the second case, for the foam laser irradiation, the maximum proton and deuteron energy is $2.5 \mathrm{MeV}$ and $3.4 \mathrm{MeV}$, respectively. The two spectra are similar because the equivalent target thickness was $1 \mathrm{mg} / \mathrm{cm}^{2}$ for the thin high density foil and $3 \mathrm{mg} / \mathrm{cm}^{2}$ for the thick low density foam. Decreasing the $\mathrm{CD}_{2}$ film thickness the proton energy and yield decrease. Moreover, using thick ( $3 \mathrm{~mm}$ ) high density polyethylene the backward accelerated protons is very low, only of the order of $200 \mathrm{keV}$. A deconvolution of SiC spectra can be performed by using Boltzmann ion energy distributions, as reported in Fig. 4, indicating the contribution of protons coming from D-D nuclear reaction and coming from the proton acceleration of absorbed hydrogen in the polymer, deuterium ion acceleration and of the six charge states of the carbon ions.

Fig. 5 shows a typical plastic scintillator TOF spectrum relative to the laser irradiation of the thick $\mathrm{CD}_{2}$ foam, $3 \mathrm{~mm}$ thick, indicating a forward prompt detection of X-rays due to plasma emission and fast electron Bremsstrahlung, used as start signal, followed by a peak signal at $110 \mathrm{~ns}$. Due to the flight distance of $2.6 \mathrm{~m}$, this peak corresponds to neutron detection at $2.45 \mathrm{MeV}$, characteristic of the neutron emission from D-D fusion events.

Fig. 6a shows a photo of one bubble neutron dosimeter (4 bubble/ $\mu \mathrm{Sv}$ sensitivity) detecting forward emitted neutrons. Generally the dosimeter response was of 1 bubble per laser shot from $10 \mu \mathrm{m}$ high density thin target and from $3 \mathrm{~mm}$ low density foam at a distance of $25 \mathrm{~cm}$ from the target. Fig. 6b shows a typical Cr-39 track detector covered with $100 \mu \mathrm{m} \mathrm{Al}$ absorber reporting typical tracks due to neutron detection after many laser shots on different $\mathrm{CD}_{2}$ targets.

The plasma characterization was performed in terms of temperature using different approaches. One was obtained 


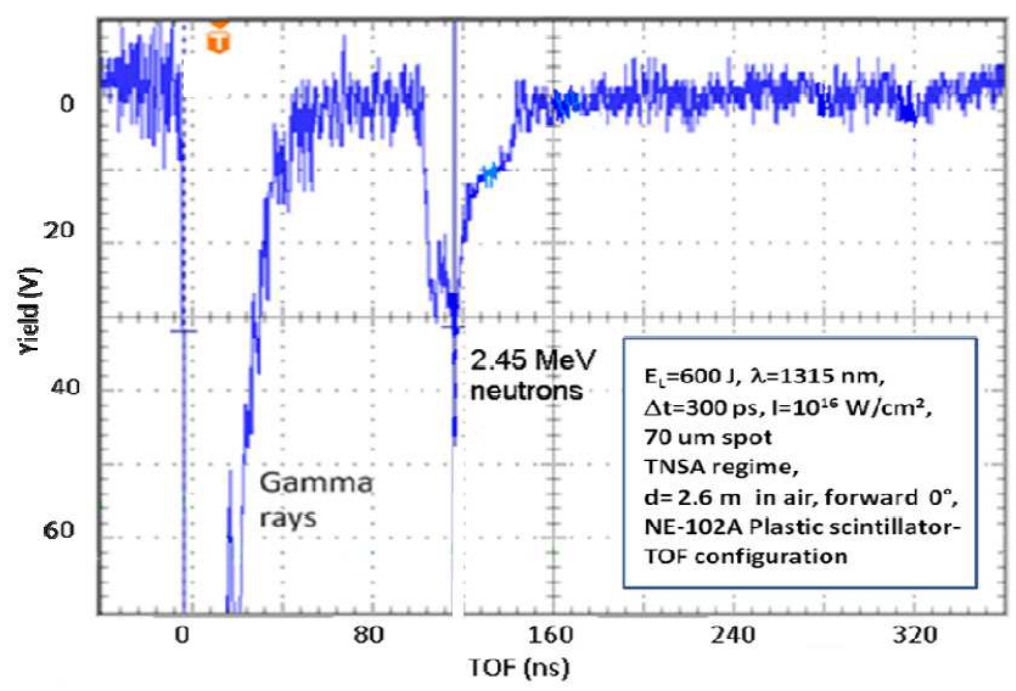

Figure 5. Deconvolution of a SiC spectrum to distinguish the different ion contributions using Boltzmann ion energy distributions.

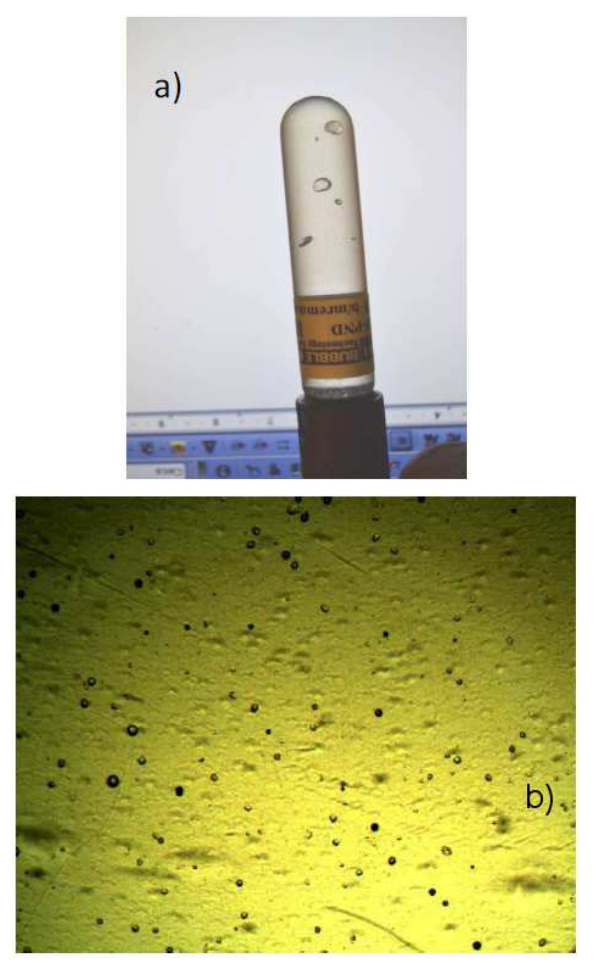

Figure 6. Neutron measurements from $\mathrm{CD}_{2}$ foam target irradiation after a lot of laser shot irradiation at $600 \mathrm{~J}$ using the bubble neutron dosimeter (a) and the Cr-39 track detector covered by $100 \mu \mathrm{m} \mathrm{Al}$ absorber (b).

from the ion deconvolution Boltzmann peaks, indicating a plasma temperature of about $6 \mathrm{keV}$. Another one was performed by measuring the adiabatic plasma expansion in vacuum through the $\mathrm{X}$-ray streak camera images, giving an expansion length of $400 \mu \mathrm{m}$ in an exposition time of $1 \mathrm{~ns}$, i.e. a velocity of $4 \times 10^{5} \mathrm{~m} / \mathrm{s}$, corresponding to a temperature of about $5 \mathrm{keV}$, in agreement with the other techniques.
This relatively low plasma temperature indicates that the high number of fusion events measured during the experiment is not due to thermal interactions, which may produce a very low reaction flux due to the low D-D cross section at these so low atoms velocities. The high number of fusion events instead is due to the high kinetic energy of accelerated deuteron ions against deuterium atoms that around $3 \mathrm{MeV}$ shows the maximum value of the D-D cross-section.

A direct and clear evidence of nuclear events is shown in spite of small efficiency of neutron detection and high background noise and of the little SiC detectors solid angle. Assuming an isotropic neutron emission from the D$\mathrm{D}$ nuclear fusion reactions and an efficiency of the order of $10^{-7}$ both for the bubble neutron dosimeters and CR39 track detectors, the results obtained by cumulating ten laser shots indicated a value of about $4 \times 10^{8}$ neutrons per laser shot. A similar result was obtained for the $\mathrm{SiC}$ detectors and ion collectors by considering the $3.0 \mathrm{MeV}$ protons current, the solid angle subtended by the detectors and assuming the angular emission to have a main contribution in forward direction with an angular aperture of about $70^{\circ}$. Such results are in agreement also with the theoretical previsions obtainable by considering the measured proton flux, the PE density and the D-D cross section at the 3.0 $\mathrm{MeV}$ deuteron kinetic energy.

The evaluation concerning the number of nuclear events is in good agreement with the values obtained in previous experiments and with literature data [12-22].

Fig. 7 reports a comparison between different literature data performed at different laser pulse energies irradiating deuterated targets and calculating the number of neutron events per laser shot. It is possible to observe that the number of events increases with the laser energy and with the laser pulse duration. Our measurements performed at $600 \mathrm{~J}$ pulse energy and $300 \mathrm{ps}$ pulse duration seems to be optimal in order to enhance the number of fusion events according to the reported literature data. 


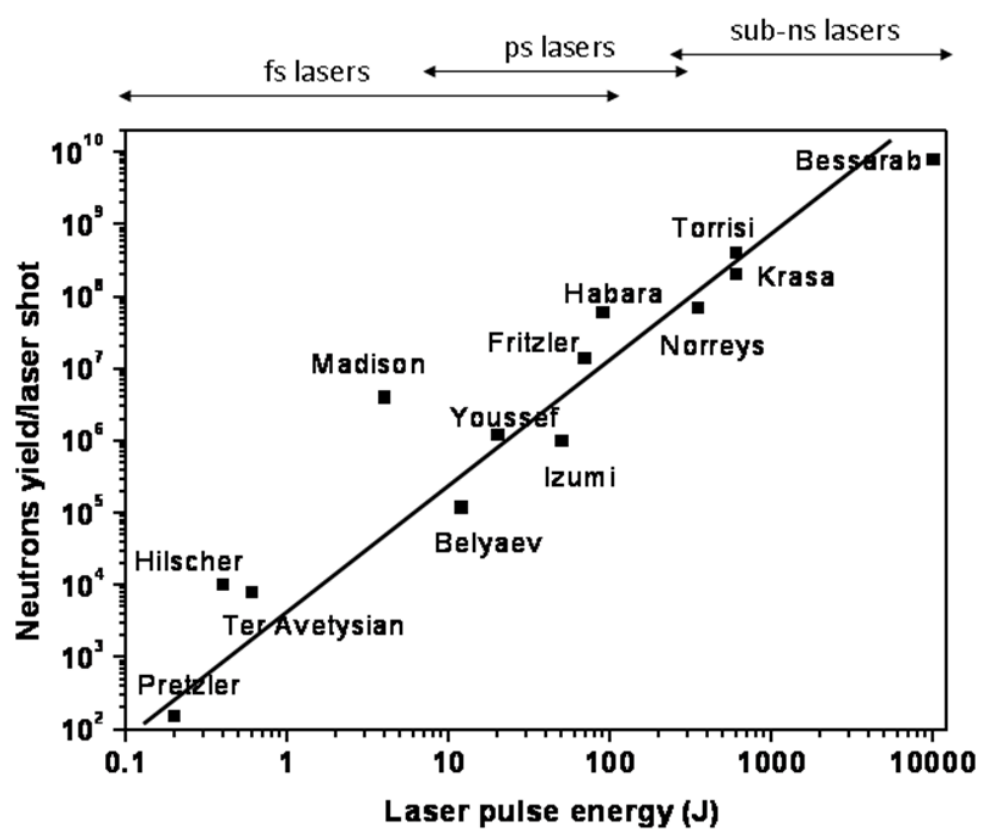

Figure 7. Neutron yield/laser shot as a function of laser pulse energy and laser pulse duration from literature data and comparison with our measurements.

\section{Discussion and conclusions}

The D-D nuclear reaction fusion can be induced in laser-generated plasma at a laser irradiance $\mathrm{I}^{2} \sim 5 \times$ $10^{16} \mathrm{~W} \mathrm{~m}^{2} / \mathrm{cm}^{2}$. The number of events can be raised by increasing the kinetic energy of the accelerated deuterium ions, of the order of 3-5 MeV, both using thin and high density deuterated PE and using thick and low density foam foils. An optimal target thickness can be that ranging between $1-3 \mathrm{mg} / \mathrm{cm}^{2}$ that permits to obtain about $4 \times 10^{8}$ characteristic neutrons and protons emission. The plasma temperature was evaluated to be of the order of 5-6 keV, too low to justify the high number of measured nuclear events that instead can be due to the high cross section of $\mathrm{MeV}$ deuteron ions interacting with other $\mathrm{D}$ atoms of the target/plasma. On this basis it is possible to imagine that other fusion processes, with comparable crosssection, such as D-T, D- ${ }^{3} \mathrm{He}$ as well as T-T, may be induced in the generated plasmas using the Asterix laser PALS facility. In conclusion, the reported results confirm usefulness the concept of using the plasma produced by sub-nanosecond pulses of TW lasers giving about 600 $\mathrm{J}$ pulse energy for generation of $\mathrm{MeV}$ protons and neutrons. In particular, the experiments conducted at PALS demonstrate that in the laser-produced plasma expanding in the forward direction with a broad angular distribution a large number of multi-MeV deuterons suitable for beamtarget experiments can be generated. Our experiments indicate that the yield of neutrons from the $\mathrm{d}(\mathrm{d}, \mathrm{n})^{3} \mathrm{He}$ reaction and $\mathrm{d}(\mathrm{d}, \mathrm{p})^{3} \mathrm{H}$ reaches up to $4 \times 10^{8}$ neutrons/shot and $4 \times 10^{8}$ protons/shot make possible the generation of about monochromatic neutron source useful for many applications.

\section{Acknowledgements}

Work supported by LASERLAB-EUROPE N. pals001823 "High energy proton acceleration from thin advanced targets at PALS". The authors thank the useful relief of Dr. A. Velyhan, Dr. M. Pfeiffer and Dr. E. Krousky from PALS and the useful suggestions given by Dr. J. Krasa from Institute of Physics of ASCR, Czech Republic during the experiments.

\section{References}

[1] Shalom Eliezer, "The interaction of high-power lasers with plasmas", Series Plasma Physics, IOP, Bristol, UK, 2002.

[2] L. Torrisi "Ion acceleration from laser-generated plasma: methods, diagnostics and applications" 12th Kudowa Summer School, "Towards Fusion Energy" Kudowa Zdrój, June 9-13, (IL-7), pg. 28-31, 2014.

[3] B. M. Hegelich et al., Nature Letters 439, 441 (2006).

[4] L. Torrisi et al., J. Appl. Phys. 91, 4685 (2002).

[5] L. Torrisi et al., Review of Scientific Instruments 83, 1 (2012).

[6] National Physical Laboratory 2014, Kaye\&Laby, Tables of Physical \& Chemical Constant, Chapter 4, Sect. 4.7, Subsect. 4.7.4 Nuclear fusion: http://www.kayelaby.npl.co.uk/atomic_and__ nuclear_physics/4_7/4_7_4.html

[7] J. Limpouch et al., Plasma Phys. Control. Fusion 46, 1831 (2004).

[8] Vector Field, "Opera-3d 16 Electromagnetics and Multiphysics Design Software", (2013) website: http://www.scientificcomputing.com/product- 
releases/2013/08/opera-3d-16-electromagnetics-andmultiphysics-design-software

[9] M. Cutroneo et al., J. Material Research 28, 87 (2012).

[10] M. Cutroneo et al., Acta Polytechnica 53,138 (2013)

[11] BTI- Bubble Technology Industries Inc. Bubble Detector Neutron Dosimetry, Technical Specifications. Available online (accessed on 20 October, 2014): http://bubbletech.ca/wpcontent/uploads/2014/02/BTI_BUBBLE_General_May720091.pdf

[12] G. Pretzler et al., Phys.Rev. E 581165 (1998).

[13] D. Hilscher et al., Phys. Rev. E 64, 016414 (2001).

[14] S. Ter-Avetisyan et al., Phys. Plasmas 12, 012702 (2005).
[15] K.W. Madison et al., Phys. Rev. A 70, 053201 (2004).

[16] V.S. Belyaev et al., Laser Phys. 16, 1647 (2006).

[17] A. Youssef,et al., Phys. Plasmas 12, 110703 (2005).

[18] N. Izumi et al., Phys. Rev. E 65, 036413 (2002).

[19] S. Fritzler et al., Phys. Rev. Lett. 89, 165004 (2002).

[20] P.A. Norreys et al., Plasma Phys. Contr. Fusion 40, 175 (1998).

[21] H. Habara et al., Phys. Rev. E 70, 046414 (2004).

[22] P.A. Norreys et al., Plasma Phys. Control. Fusion 47, L49 (2005).

[23] J. Krasa, et al., Laser and Particle Beams, 1 (2013).

[24] A.V. Bessarab et al., Sov. Phys. JETP. 75, 970 (1992). 